neuronal markers by immunofluorescence and quantitative PCR(qPCR).

Methods Human bowel was collected following a pull-through procedure in four patients with short segment HSCR. Protein expression of ENS and neuronal markers (p75, SOX10, PHOX2b, Hu and GLUT1) in ganglionic, TZ and aganglionic region of the bowel were visualised through immunofluorescence and mRNA levels of the corresponding markers were quantified using qPCR.

Results Immunofluorescence analysis showed a gradual loss of SOX10, PHOX2b and $\mathrm{Hu}$ protein in the lower TZ and absence in the aganglionic region. However, generally the marker expression presented with inter-patient variability within the TZ. By contrast, GLUT1 was highly expressed in the perineurium of thickened nerve trunks, characteristic of the aganglionic region. Perineurial structures positive for GLUT1 were also visualised in the TZ, but to a lesser extent than the aganglionic region. This observation corresponded with a decrease in mRNA levels of p75, SOX10, PHOX2b and $\mathrm{Hu}$ from the ganglionic to the aganglionic region and an increase in GLUT1.

Conclusion This work displayed a trend of decreasing number of cells expressing ENS and neuronal markers from the ganglionic to aganglionic region, through the TZ. However, our analysis reveals inter-patient variability in the cellular composition of HSCR bowel, especially in the TZ. Possibly explaining the variable functional outcome for HSCR children with TZ pull-through.

\section{G325(P) A SINGLE-CENTRE AUDIT EVALUATING THE APPROPRIATE USE OF DMSAS FOR THE INVESTIGATION OF PAEDIATRIC UTI}

S Ramachandran, I Hasan, P Set. Radiology Department, Cambridge University Hospitals NHS FT, Cambridge, UK

\subsection{6/archdischild-2020-rcpch.281}

Aims Technetium-99m-labeled dimercaptosuccinic acid (DMSA) scintigraphy represents an important imaging modality in investigating select paediatric patients with urinary tract infection (UTI). Recently updated NICE guidelines outline the indications for DMSA according to age group. This audit aimed to assess adherence to these guidelines and evaluate their reliability.

Methods Using PACS, we retrospectively evaluated all firsttime DMSAs performed at a tertiary centre to investigate paediatric UTI over a period of 24 months. Data collected included patient age, clinical justification, timing of the scan and abnormalities reported.

Results 105 patients underwent DMSAs (mean age: 4.0 years, range: 4 months-15.6 years). Of these, 85 cases $(81.0 \%)$ adhered to NICE guidelines. Of the 20 non-adherent cases, 8 $(40 \%)$ misinterpreted the definition of recurrent UTIs, 8 (40\%) were performed inappropriately after a solitary UTI, and $4(20 \%)$ misinterpreted the definition of an atypical UTI. Adherence was poorest for patients under 6 months at $71.4 \%$. NICE guidelines had a $88.9 \%$ sensitivity $(95 \%$ CI $65.3-$ 98.6\%); 20.7\% specificity (95\% CI $12.8 \%-30.7 \%), \quad 18.8 \%$ PPV (95\% CI 16.0\%-22.0\%); and 90\% NPV (95\% CI 69.697.3\%).

Conclusion We demonstrate relatively consistent adherence to NICE guidelines with some room for improvement. We advocate improving awareness amongst referring clinicians on the guidelines, particularly for the youngest age group and on what constitutes a recurrent or atypical UTI. Improving adherence ensures that unnecessary DMSAs are avoided, precluding radiation exposure and emotional stress. Although limited by our small sample size, we show good sensitivity but poor specificity of NICE guidelines.

\section{G326(P) INVESTIGATION AND MANAGEMENT OF PAEDIATRIC UROLITHIASIS - A UK SINGLE CENTRE EXPERIENCE}

${ }^{1}$ A Duncanson, ${ }^{2} S$ Hotonu. 'Paediatric Nephrology, Great North Childrens' Hospital, Newcastle Upon Tyne, UK; ${ }^{2}$ Paediatric Urology, Great North Childrens' Hospital, Newcastle Upon Tyne, UK

\subsection{6/archdischild-2020-rcpch.282}

Aim To evaluate our centre's experience on the management of urinary tract stones in children according to current European Association of Urology (EAU) guidelines.

Methods A retrospective review of all cases of urolithiasis treated in our tertiary paediatric centre. This encompasses a 6 year period (June 2013- May 2019). Data collected included patient demographics, urinary and biochemical workup, stone burden and analysis, surgical management, outcomes of treatment and follow up. Compliance with EAU guidelines was also audited.

Results Thirty seven patients were identified (22 males). Median age was 8 years (range 1-16 years). Mean weight was $31.5 \pm 8$ kilograms, with 3 patients being above the 75 th centile. Risk factors for stone formation were identified in twenty four patients: four patients (10.8\%) were on medication known to precipitate urolithiasis, two patients $(5.4 \%)$ had a family history of renal stones, five patients $(13.5 \%)$ had a metabolic cause, ten patients $(27 \%)$ had a history of recurrent urinary tract infections and three patients $(8.1 \%)$ had a combination of risk factors. The remaining thirteen patients were idiopathic stone formers. Nine patients had bilateral renal stones, twenty five unilateral, and three had bladder stones. Majority of stones were calcium based. Twenty two patients $(59.4 \%)$ had surgical management of stones, with 3 patients requiring repeat procedures for high stone burden. Eight patients $(21.6 \%)$ had impairment of renal function secondary to stones.

Conclusions Management of paediatric urolithiasis is complex and is associated with significant morbidity. The majority of patients have clear risk factors for stone formation. Investigation according to European guidelines provides the means to identify such patients. Further multicentre collaborative studies are warranted.

\section{G327(P) AN AUDIT OF ACUTE KIDNEY INJURY IN CHILDREN}

${ }^{1} \mathrm{R}$ Collier, ${ }^{2} \mathrm{R}$ Holt, ${ }^{2} \mathrm{CA}$ Jones, ${ }^{3} \mathrm{~S} J \mathrm{McW}$ illiam. ${ }^{1}$ Medical School, University of Liverpool, Liverpool, UK; ${ }^{2}$ Department of Nephrology, Alder Hey Children's NHS FT, Liverpool, UK; ${ }^{3}$ Department of Women's and Children's Health, University of Liverpool, Liverpool, UK

\subsection{6/archdischild-2020-rcpch.283}

Aims Acute Kidney Injury (AKI) in hospitalised children is significantly associated with increased morbidity and mortality. The British Association of Paediatric Nephrology AKI management guidelines recommend early identification of patients at 
high risk for AKI to enable preventative measures to be implemented. This retrospective audit aimed to describe the epidemiology of AKI at a tertiary paediatric centre in order to prioritise interventions.

Methods All inpatients with an AKI stage 2 or 3 alert between 1st March and 31st May 2017 were included in this audit. The electronic healthcare record for each patient was accessed and data extracted.

Results Over a 3 month period there were 354 AKI stage 2 or 3 alerts accounting for 125 AKI episodes affecting a total of 101 patients. Males (53.5\%) and females (46.5\%) were evenly represented. Children aged between 1 month and 2 years were most frequently affected (37\%). 22\% of patients were under the care of cardiology, $12 \%$ under general paediatrics, and $11 \%$ under respiratory. The majority of episodes lasted only 1 day, with 55 episodes (44\%) lasting for 2 or more days. $51 \%$ of AKI episodes were associated with exposure to at least one nephrotoxic medication during, or in the 7 days preceding, the event. The most common medications were furosemide (14\%), piperacillin with tazobactam (14\%) and ibuprofen (11\%). Overall the cause of AKI was infrequently documented ( $37 \%$ of episodes), however, where documented, the leading causes were cardiac surgery $(28 \%)$ and nephrotoxic medications (20\%).

Conclusion Cardiac surgery represents a significant risk factor for the development of AKI in children. In addition, over half of children who developed AKI were exposed to nephrotoxic medications, and this was the second most common identified cause. Future AKI management strategies should focus on delivering improvements for these groups of patients to reduce the impact of AKI.

\section{G328(P) ABSTRACT WITHDRAWN}

\section{G329(P) INCIDENCE OF AMPHOTERICIN-INDUCED ACUTE KIDNEY INJURY IN A PAEDIATRIC POPULATION: A SYSTEMATIC REVIEW}

${ }^{1} \mathrm{D}$ Mistry, ${ }^{2,3} \mathrm{~L}$ Oni, ${ }^{3,4}{ }^{\mathrm{S} J} \mathrm{McW}$ Milliam. ${ }^{1}$ Medical School, University of Liverpool, Liverpool, UK; ${ }^{2}$ Department of Paediatric Nephrology, Alder Hey Children's NHS FT, Liverpool, UK; ${ }^{3}$ Department of Women's and Children's Health, University of Liverpool, Liverpool, UK; ${ }^{4}$ MRC Centre for Drug Safety Science, University of Liverpool, Liverpool, UK

\subsection{6/archdischild-2020-rcpch.284}

Aims The aim of this project was to perform a systematic literature review to determine the incidence of Amphotericininduced Acute Kidney Injury (AKI) in the paediatric population.

Methods A systematic review was performed to obtain articles until October 2018 using Scopus and Medline, with additional articles obtained using the bibliographies from selected studies. Studies were chosen if they included an intervention of Amphotericin with monitoring of renal function and a declaration of how AKI would be defined. All types of studies were considered and those retrievable in full text and English were included.

Results Of sixty-three articles identified, seventeen were available in full text. A further ten studies were rejected due to not mentioning dose, duration or outcomes. The remaining seven studies totalled 279 patients aging between
0-15 years. The ratio of boys to girls was 50:50 (demographic data reported in only 4 of 7 studies). Of the seven studies none were randomised controlled trials. Visceral Leishmaniasis was the indication for treatment with liposomal Amphotericin in 6 of the 7 studies. The dose of Amphotericin ranged from $15-30 \mathrm{mg} / \mathrm{kg}$ and a duration of 2 - 21 days. The total incidence of AKI was found to be $1 \%$, with the pRIFLE criteria being the most commonly used method of defining AKI, however many of the studies did not explicitly define AKI in their outcome criteria, leading to low identification rates. The optimal dose range is 3-4 $\mathrm{mg} / \mathrm{kg}$ for a regime of 5-day treatment followed by a further dose on day 10. Additional outcomes included risk factors for AKI with underlying infection being the most consistent.

Conclusion This systematic review found an incidence of Amphotericin-induced AKI in a paediatric population of $1 \%$. Comparatively, adults show an incidence rate of around 50\%, suggesting large differences between the two cohorts. There were limitations to this study, mainly due to the lack of standardisation in the definition of drug-induced AKI in children across the studies. Improved reporting of Amphotericininduced AKI, using standardised AKI definitions, is required to confirm the true incidence in children.

\section{G330(P) A SYSTEMATIC REVIEW OF THE RISK FACTORS ASSOCIATED WITH VANCOMYCIN-INDUCED ACUTE KIDNEY INJURY IN CHILDREN}

${ }^{1} \mathrm{C}$ Williams, ${ }^{2,3} \mathrm{~L}$ Oni, ${ }^{3,4} \mathrm{SJ}$ McWilliam. ${ }^{1}$ Medical School, University of Liverpool, Liverpool, UK; ${ }^{2}$ Department of Paediatric Nephrology, Alder Hey Children's NHS FT, Liverpool, UK; ${ }^{3}$ Department of Women's and Children's Health, University of Liverpool, Liverpool, UK; ${ }^{4}$ MRC Centre for Drug Safety Science, University of Liverpool, Liverpool, UK

\subsection{6/archdischild-2020-rcpch.285}

Aims Vancomycin has been suggested to contribute to acute kidney injury in adults, however few studies have addressed its role in the paediatric population. The primary aim of this paper is to review the evidence surrounding the risk factors involved in the development of paediatric acute kidney injury as a result of vancomycin administration.

Methods A systematic search was performed in November 2018 on PubMed, Web of Science and Scopus using ((AKI OR acute kidney injury OR nephrotoxic* OR renal injur* OR renal insufficiency OR kidney damage) AND (vancomycin OR vancocin) AND ( $p$ *ediatric OR child* OR infant* OR child* OR adolescen* OR neonat*)). Eligible studies were meta-analyses, clinical trials, observational studies or case series of vancomycin use in a paediatric population (0-18 years) reporting outcomes of nephrotoxicity.

Results Of 1021 records identified, sixteen retrospective cohort studies were eligible for inclusion in the review, totalling 10,575 patients with an overall vancomycin-induced acute kidney injury (v-AKI) incidence of $13.3 \%$. Thirteen studies considered the effect of co-administration of vancomycin with other nephrotoxins. Use of concomitant nephrotoxic medications was associated with a significantly increased risk of AKI. Co-administration of aminoglycosides, piperacillin-tazobactam, vasopressors, furosemide (and other loop diuretics), ACE inhibitors and NSAIDs were all found to be significantly associated with increased risk of AKI in at least one study. However, there was significant variation between studies. Six 